\title{
CARBOHYDRATE METABOLISM-A REVIEW
}

\author{
Azmy R. Boutros, M.B., CH.B., D.A., F.F.A.R.c.s.*
}

CARBOHYDRATE metabolism has been the subject of extensive research during the last decade. ${ }^{1-6}$ The possibility that induction of the anaesthetic state may be due to some derangement in cellular metabolism makes the anaesthetist take more than the usual academic interest in the subject.

Details of the important achievements in this field are beyond the scope of this review and the reviewer will endeavour to discuss the more salient points of the subject without entering into too much detail.

The functions of carbohydrate metabolism are more involved than was previously thought and, in short, they consist of the following:

1. Energy production. The process of glucose oxidation consists of a series of reactions, the large majority of which are exergonic, that is to say, these reactions produce energy, which is made available to the organism for the performance of various functions, thus:

(a) Energy in the form of heat may be used to maintain the body temperature, and is thus lost to the body.

(b) Energy may be immediately transferred to another type of chemical biological reaction that requires energy for its completion (endergonic reactions).

(c) The most important outlet of the free energy produced in glycolytic reactions, howeyer, is the use of this energy in the synthesis of high-energy substances, which can subsequently decompose, releasing this locked energy when and where it is required. This is an extremely economical and flexible way of dealing with free energy. Broadly speaking energetic substances are of two types: (i) hydrogenated substances such as reduced diphosphopyridine nucleotide "DPNH"; (ii) phosphorylated compounds such as adenosine triphosphate "ATP" and creatine phosphate.

2. Apart from energy production, carbohydrate metabolism can supply the organism with certain necessary substances that are either unavailable to the body in the diet or present in insufficient amounts; thus some of the substances produced in the process of glycolysis provide intermediates for fatty acid synthesis (glycerophosphates and acetyl carbohydrates) and for synthesis of amino acids (pyruvate, oxalacetate, and alpha-ketoglutarate).

\section{Pathways of Carbohydrate Catabolism}

The first step in the oxidation of blood glucose is the transport of this glucose into the cells. In liver cells, this occurs by simple diffusion of the sugar through the cell membrane, ${ }^{7}$ but in other tissues and particularly in muscle cell, evidence has accumulated indicating that insulin plays an important role in facilitating the transfer of sugar through the cell membranes. ${ }^{8.9}$

\footnotetext{
*Assistant Professor, Department of Anaesthesia, University of Saskatchewan, Saskatoor, Saskatchewan.
}

Can. Anaes. Soc. J., vol. 9, no. 4, July, 1962 
Following entry into the cells, glucose is rapidly incorporated into the cellular metabolic machinery by a process of phosphorylation (Step 1, Fig. 1). Thus, with the aid of the hexokinase enzyme and a phosphate molecule from adenosine triphosphate (ATP), glucose is changed into glucose-6-phosphate and the ATP is changed into ADP (adenosine diphosphate).

Some workers ${ }^{1}$ think that insulin has no effect on the hexokinase reaction and the phosphorylation of glucose, while others ${ }^{10}$ feel that insulin facilitates the transport of glucose into the cell, as well as the subsequent phosphorylation process.

Glucose-6-phosphate is an important branch point in the subsequent catabolic processes of carbohydrates via the Embden-Meyerhof pathway or the pentose phosphate pathway, the so-called hexose monophosphate shunt.

Conversely glucose-6-phosphate may be reconverted into glucose. This reaction occurs only in the liver under the influence of the enzyme glucose-6-phosphatase with energy production, which appears in the form of heat. Glucose-6-phosphate may also form glycogen through a process of assimilation.

This review will deal only with the general outlines of these processes, and the interested reader seeking more detailed information is referred to a number of excellent articles and reviews where the subject is discussed in great detail. ${ }^{1-5}$

\section{Glycolytic Pathways}

The complete dissimilation of glucose to carbon dioxide and water. occurs in two phases:

A. The Embden-Meyerhoff phase, which can function under anaerobic conditions. Through this phase, one molecule of glucose is changed in to two molecules of pyruvic acid or two molecules of lactic acid under anaerobic conditions.

$B$. The citric acid or Krebs cycle phase, which is obligatory aerobic and cannot function under anaerobic conditions. Through this phase pyruvic acid is changed into carbon dioxide and water.

\section{A. The Embden-Meyerhof Pathway ${ }^{1,11}$ (Fig. 1)}

This is the main pathway of carbohydrate catabolism and, in the brain and skeletal muscle cells, the only one. In liver, adipose tissue, and red blood cells, however, it accounts for a variable fraction of the glucose catabolized, the rest of the glucose being catabolized through the pentose phosphate pathway, or the hexose monophosphate shunt, which will be discussed later.

A summary of the steps involved in the Embden-Meyerhof pathway is as follows:

1. Glucose-6-phosphate to fructose-6-phosphate (Step 2). Process: isomerization. Enzyme: phosphohexose isomerase.

2. Fructose-6-phosphate to fructose-1,6-phosphate (Step 3). Process: phosphorylation. ATP supplies the extra high-energy phosphate molecule, thus raising the energy of the compound and rendering the reaction irreversible. Enzyme: phosphofructokinase.

3. Fructose-1,6-phosphate to two molecules of triose phosphate (Step 4). Fructose-1,6-phosphate is split into two triose phosphate molecules (2 dehydroxyacetone) each singly phosphorylated. Enzyme: aldolase (zymohexase). 


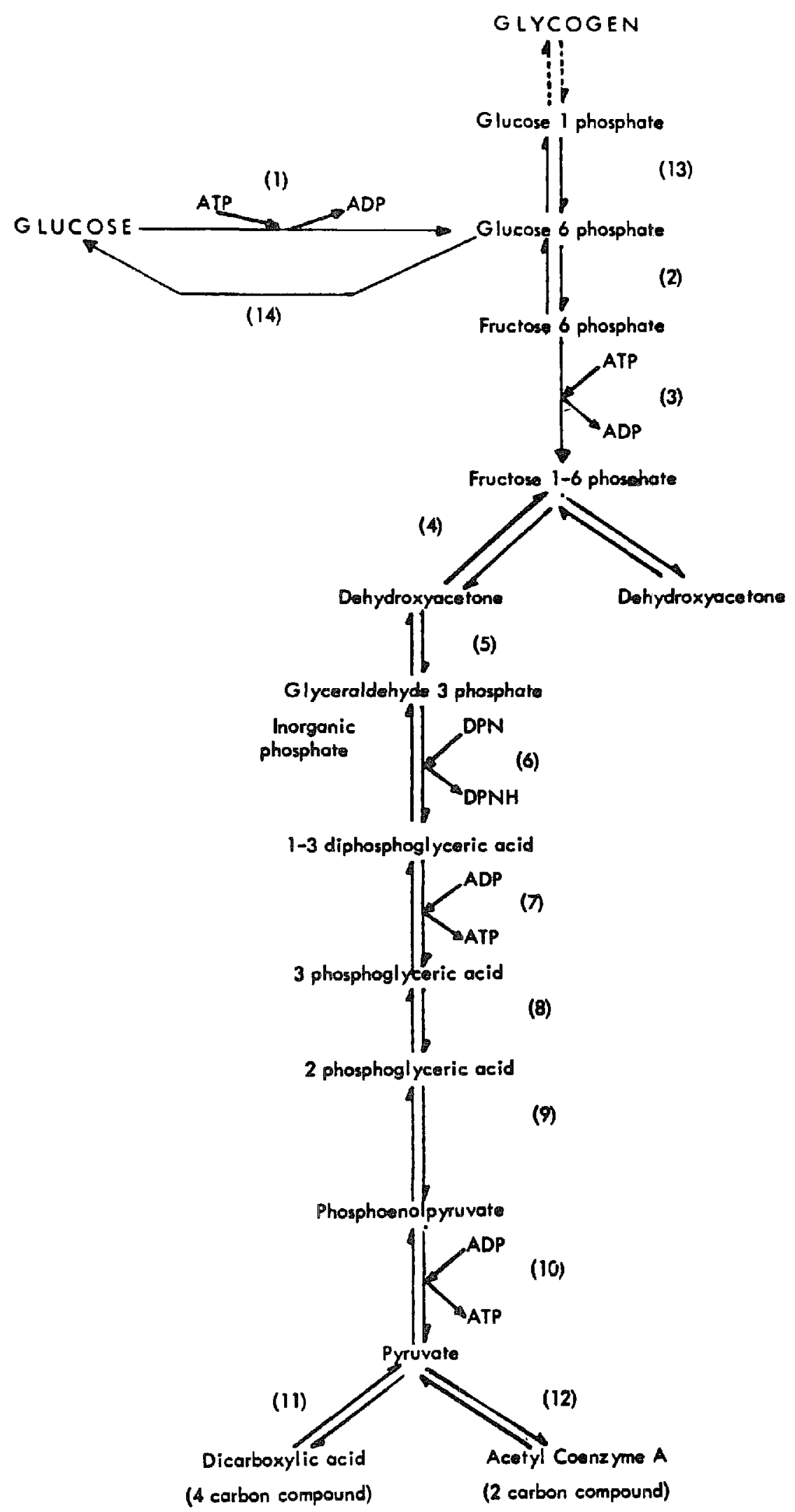

Figure 1. Carbohydrate metabolism. The Embden-Meyerhof Pathway. 
4. Two dehydroxyacetone to two glyceraldehyde-3-phosphate (another triose phosphate) (Step 5). Enzyme: phosphotriose isomerase.

5. Two glyceraldehyde-3-phosphate to two 1,3-diphosphoglyceric acid (Step 6). Enzyme: glyceraldehyde-3-phosphate dehydrogenase inhibited by iodoacetate. This is an important reaction in the process of glycolysis and involves the following:

(a) Oxidation by dehydrogenation. The hydrogen is picked up by oxidized diphosphopyridine nucleotide (DPN), which acts as a hydrogen carrier and becomes reduced to DPNH $+\mathrm{H}$. Regeneration of the DPNH is achieved in one of two ways: (i) DPNH can be reoxidized by giving its hydrogen to the riboflavincytochrome hydrogen transfer system and ultimately to molecular oxygen forming water. Obviously this can only occur under aerobolic conditions. (ii) Under anaerobic or relatively anaerobic conditions DPNH can give its hydrogen to pyruvic acid reducing it to lactic acid. In this case pyruvic acid will act as the hydrogen acceptor, thus leaving DPN oxidized and able to participate again in the oxidation of glyceraldehyde to glyceric acid. As will be explained later, all the energy production from the dissimilation of glucose to pyruvic acid occurs in Steps 6, 7, and 10; hence the importance of regenerating DPN. When aerobic conditions are restored, lactic acid will give the hydrogen to DPN and becomes oxidized to pyruvic acid.

(b) Phosphorylysis or the addition of inorganic phosphoric acid. It should be noted that glutathione acts as prosthetic group for the enzyme glyceraldehyde-3phosphate dehydrogenase and that the $\mathrm{SH}$ group on the dehydrogenase actually participates in the process of oxidation of the glyceraldehyde. ${ }^{12}$

6. Two 1,3-diphosphoglyceric acid to two 3-phosphyglyceric acid (Step 7). This is an exergonic reaction, the energy released helping to build up two molecules of ATP from ADP (adenosine diphosphate) leaving behind 3-phosphoglyceric acid. Enzyme: phosphoglyceric kinase.

7. Two 3-phosphoglyceric acid to two 2-phosphoglyceric acid (Step 8). Enzyme: phosphoglyceromutase.

8. Two 2-phosphoglyceric acid to two phosphoenolpyruvic acid (Step 9). Process: dehydration. Enzyme: enolase, inhibited by fluoride ion; hence the addition of flouride salts to the anticoagulant mixture added to blood collected for blood sugar estimation, to inhibit glycolysis.

9. Two phosphoenolpyruvic acid to two pyruvic acid (Step 10). This is a dephosphorylation exergonic reaction supplying two high-energy phosphate bonds to two molecules of ADP to form two molecules of ATP.

\section{THE ENERGY VALUE OF THE EMBDEN-MEYERHOF REACTION}

As was mentioned before, energetic compounds are either in the form of highenergy phosphate bonds added to ADP to produce ATP or in the form of reduced diphosphopyridine nucleotide (DPNH), each molecule of which, when picked up by the riboflavine cytochrome hydrogen transfer system, produces energy, which is used to build up three molecules of adenosine triphosphate from ADP. It is important to realize that if the reduced DPNH were to give its hydrogen to molecular oxygen without the intervention of the electron transfer system, the 
reaction between hydrogen and oxygen would be so explosive as to be of no value to cells, as far as energy production is concerned. Instead, the reaction takes place in a slower stepwise manner, which enables the cell to make use of the energy produced in building high-energy phosphate bonds.

The dissimilation of one molecule of glucose to pyruvic acid will produce two high-energy bonds in Step 7 and two more in Step 10. These four energy bonds appear in the form of four molecules of ATP. The reduction of two DPN molecules to DPNH in Step 6, and their subsequent incorporation in the hydrogen transfer system, produces energy equal to six high-energy phosphate bonds, which are also used to build up six new molecules of ATP.

Thus the total output of energy is ten high-energy phosphate bonds. However, as one molecule of ATP is used in Step 1 (phosphorylation of glucose) and another molecule is used in Step 3 (phosphorylation of fructose-6-phosphate), the net energy output of the reaction glucose to pyruvic acid is eight high-energy bonds (eight molecules of ATP).

FATE OF PYRUVIC ACID

Pyruvic acid is a very important branch point in carbohydrate metabolism, thus:

1. Pyruvic acid may be converted to glucose or glycogen by reversal of the Embden-Meyerhof pathway.

2. Through a process of transamination pyruvic acid can give rise to 1-alanine.

3. Pyruvic acid can join the citric acid cycle as acetyl coenzyme $A$ or a dicarboxylic acid (oxalacetic or malic) to be completely dissimilated to $\mathrm{CO}_{2}$ and $\mathrm{H}_{2} \mathrm{O}$.

4. Pyruvic acid can be reduced to lactic acid thus regenerating the DPNH to DPN to be used in Step 6 (Fig. 1) as was mentioned before.

5. Pyruvic acid can be converted to a lipid.

B. The Citric Acid or Krebs Cycle (Fig. 2)

This is the final pathway through which glucose goes to become completely dissimilated to carbon dioxide and water. This cycle is by obligation aerobic and cannot function in the absence of oxygen.

It must be emphasized that this series of reactions actually forms a common pathway for the final oxidation of fatty acids and of amino acids, as well as of the products of glycolysis. The starting point of the Krebs, or tricarboxylic acid, cycle is the combination of acetyl coenzyme $A$, or active acetate, and oxaloacetic (dicarboxylic) acid to form citric acid (tricarboxylic acid), and it is very important to have a clear idea of the mode of formation of these two main substrates.

1. Acetyl coenzyme A (active acetate). (a) Acetyl coenzyme A is produced by a process of oxidative decarboxylation of pyruvic acid (Fig. 2, Step 1). This reaction is irreversible and is catalyzed by a decarboxylase, which requires lipothiomide pyrophosphate as a coenzyme. The result is a loss of one molecule of $\mathrm{CO}_{2}$ and oxidation by dehydrogenation, the hydrogen being accepted by DPN to form DPNH. (b) Acetyl coenzyme A is also produced from the catabolism of fatty acids (Fig. 2, Step 13). (c) It can also be produced to some extent from ketogenic amino acids. 


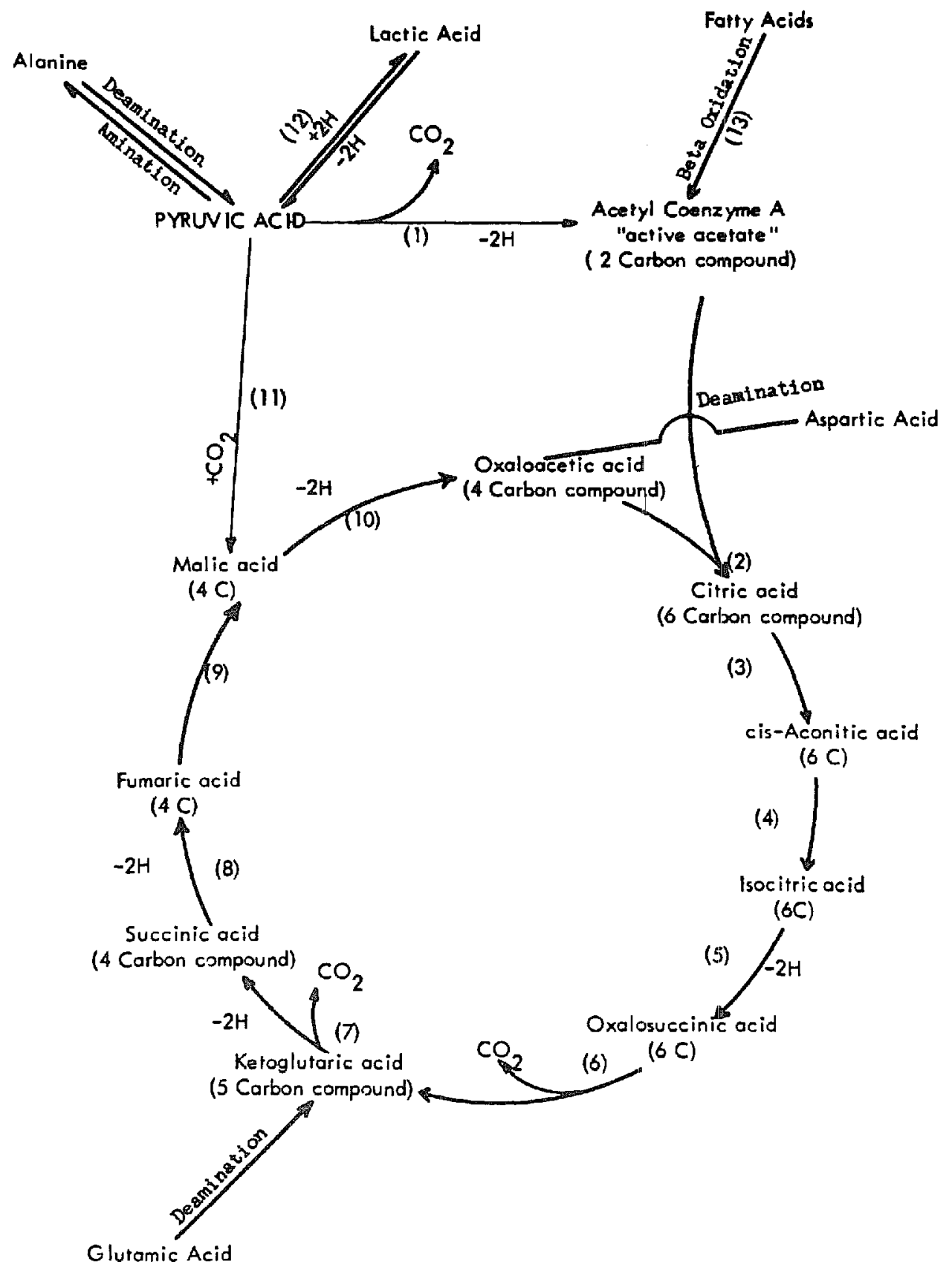

Figure 2 Carbohydrate metabolism. The Citric Acid Cycle.

2. Dicarboxylic actd. The dicarboxylic acid that combines with acetyl coenzyme A is actually oxaloacetic acid, which can be produced in the following ways: (a) Pyruvic acid is first converted to malic acid with the aid of the malic enzyme. This reaction entails carboxylation and reduction of the pyruvic acid (Step 11). Then matic acid is converted into oxaloacetic acid, the enzyme being malic acid dehydrogenase (Step 10). (b) Oxaloacetic acid can be directly formed from enolphosphopyruvic acid by a process of carboxylation. (c) It can be obtained as the product of the previous turn of the citric acid cycle. (d) Oxaloacetic acid can also be formed by deamination of a glucogenic amino acid (aspartic acid). 
The continuous formation of oxaloacetic acid from pyruvic acid (or enolphosphopyruvic acid) is of great importance in the maintenance of the Krebs cycle, because although oxaloacetic acid is formed from each complete turn of the cycle, yet this is by no means entirely efficient and some losses of this compound do occur.

The statement that fat burns in the flame of carbohydrate is quite accurate because whereas pyruvic acid can produce both acetyl coenzyme $A$ and oxaloacetic acid, fatty acid can only produce acetyl coenzyme $A$ (active aceta te) and thus requires a constant supply of oxaloacetic acid from some other source (mainly glycolytic processes) in order to be able to join the citric acid cycle and become completely oxidized to carbon dioxide and water. If, on the other hand, carbohydrate metabolism is deficient (as in diabetes) the products of fatty acid oxidation will be unable to join the citric acid cycle and thus accumulate as active acetate (or actually as keto acids), until the process of glycolysis is resumed and enough oxaloacetic acid is supplied.

PRODUCTS OF THE CITRIC ACID CYCLE

With each complete turn, the citric acid cycle produces the following:

1. Three molecules of carbon dioxide (Steps 1, 6, and 7). Thus it can be said that three carbon atoms of the pyruvic acid molecule were completely oxidized.

2 . Five pairs of hydrogen atoms (Step $1,5,7,8$, and 10), which eventually combine with molecular oxygen producing five molecules of water. These molecules of water are used up in the cycle for hydration processes.

3. Energy; the complete oxidation of one molecule of pyruvic acid produces 15 high-energy bonds, the breakdown of which is as follows: (a) Step 1 produces three high-energy bonds through supplying one molecule of DPNH to the cytochrome transfer system. (b) Step 5 produces three high-energy bonds-through supplying one molecule of TPNH to the cytochrome system. (c) Step 7 produces four high-energy bonds, one at the substrate level and three through supplying one molecule of DPNH to the cytochrome system. (d) Step 8 produces two high-energy bonds (oxidative through flavo-proteins). (e) Step 10 produces three high-energy bonds through supplying one molecule of DPNH to the cytochrome system.

It can thus be concluded that the complete dissimilation of one molecule of glucose would produce 38 high-energy bonds net of energy (eight bonds from the reaction glucose to two pyruvic acids, and 30 high-energy bonds from the complete oxidation of two molecules of pyruvic acid).

4. The citric àcid cycle also produces substrates that can be converted into other materials, e.g.: (a) Oxaloacetic acid can be transaminated to aspartic acid. (b) Alpha ketoglutaric acid to glutamic acid (transamination).

Both aspartic and glutamic acids can be used in the synthesis of proteins, as well as purines and pyramidenes, and in the formation of the urea Krebs cycle, and so on.

ROLE OF VITAMINS IN CARBOHYDRATE METABOLISM

(a) Niacin, in diphosphopyridine nucleotide and triphosphopyridine nucleotide (DPN and TPN), and riboflavin act as hydrogen acceptors in the transfer system, and are thus essential for the oxidative processes. 
(b) Thiamine, lipoic acid, and pantothenic acid are important coenzymes for the process of decarboxylation of pyruvic acid to active acetate, and of ketoglutaric acid to succinic acid.

(c) Biokin also plays a part in the carboxylation of pyruvic acid to give oxaloacetic acid.

\section{The Pentose Phosphate Pathway of Carbohydrate Oxidation (Fig. 3)}

The discovery, of an alternative pathway for carbohydrate catabolism (the pentose phosphate pathway or hexose monophosphate shunt) constitutes one of the most important advances in our knowledge of carbohydrate metabolism.

In brief, glucose-6-phosphate is oxidized to 6-phosphogluconic acid, the enzyme being glucose-6-phosphate dehydrogenase (Step 1). Then 6-phosphogluconic acid is further oxidized into 3-ketogluconic-6-phosphate (Step 2), the reaction being catalyzed by 6 -phosphogluconic dehydrogenase. The next step is a decarboxylation reaction in which 3-ketogluconic acid-6-phosphate is converted into ribulose-5-phosphate (Step 3).

The subsequent stages of the pentose phosphate pathway are non-oxidative and involve the interconversion of ribulose-5-phosphate to ribose-5-phosphate (Step 5), which can combine with a two-carbon moiety from xyloluse-5-phosphate to form a seven-carbon sugar sedoheptulose-7-phosphate (transketolation), the reaction being catalyzed by the enzyme transketolase, which requires thiamine as a cofactor. Glyceraldehyde phosphate is left behind after removal of the twocabon moiety from xylulose-5-phosphate (Step 6). Sedoheptulose-7-phosphate then reacts with glyceraldehyde phosphate producing erythrose-4-phosphate (a four-carbon sugar) and fructose-6-phosphate (Step 7); the latter is then changed into glucose-6-phosphate (Step 8). Fructose-6-phosphate may also be produced by a more direct pathway by a combination of xylulose-5-phosphate and ery throse4-phosphate producing fructose-6-phosphate and glyceraldehyde phosphate.

SIGNIFICANCE OF THE PENTOSE PHOSPHATE PATHWAY

1. One molecule of glucose is converted into carbon dioxide for every six molecules of glucose that enter this pathway.

2. The pathway results in the formation of ribose-5-phosphate, which is a constituent of a number of important cellular components such as nucleic acid, pyridine nucleotides, adenosine triphosphate.

3. Probably the most significant role of the pentose phosphate pathway is that it seems to be the main source of reduced triphosphopyridine nucleotide, which is an essential factor in the following process: (a) Reductive carboxylation of pyruvate to malate. (b) Conversion of carbohydrate to proteins. (c) Steroid synthesis and ascorbic acid synthesis. (d) Fatty acid synthesis-may be dependent on it. This could explain the block in fatty acid synthess -in diabetes, where the pentose phosphate pathway and TPNH . production are depressed..$^{13.14}$ However, it was recently found that acetyl coenzyme A dehydrogenase activity (which is depressed in livers of alloxan diabetic rats), and not TPNH, limits the rate of fatty acid synthesis in diabetic rats. ${ }^{15}$ (e) Several reactions involved in the metabolism of drugs. (f) Maintenance of integrity of red blood corpuscles. ${ }^{16}$ 


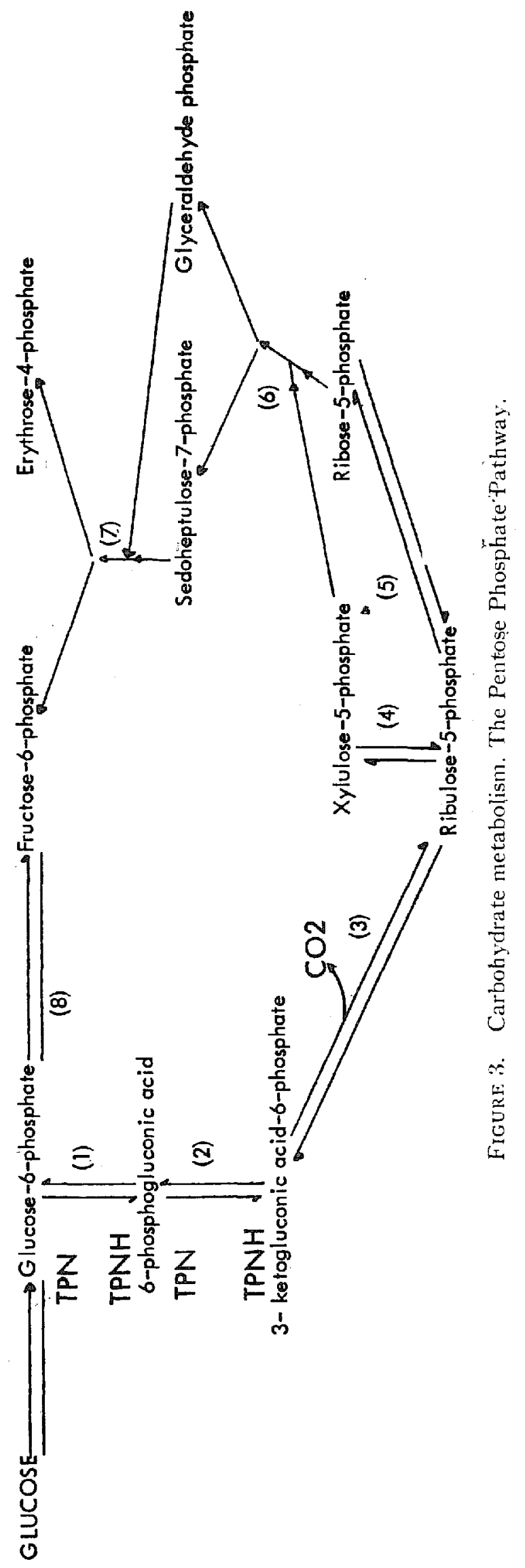




\section{Regulation of Bloqd Sugar}

The fasting blood sugar level varies between 70 and $100 \mathrm{mg}$. per $100 \mathrm{cc}$. of blood. After a carbohydrate meal, the blood sugar goes up to about 120 to $130 \mathrm{mg}$. per $100 \mathrm{cc}$. and then rapidly declines back to normal fasting levels by the end of 90 minutes to two hours. Between meals, the blood sugar level remains constant despite the continuous removal of sugar from the blood for metabolic requirements. It is thus obvious that the mechanism responsible for the maintenance of the blood sugar level is a very fine and sensitive one.

Broadly speaking, blood sugar levels depend on the following factors:

A. Addition of glucose to the blood. This consists of: (1) absorption of sugars' from the intestinal tract; (2) breakdown of glycogen to glucose (glycogenolysis); (3) conversion of non-carbohydrate elements, mainly glucogenic amino acids, into glucose (neoglucogenesis or gluconeogenesis).

B. Removal of glucose from the blood. This consists of: (1) conversion of blood glucose to glycogen (glycogenesis); (2) conversion of carbohydrates into proteins and fats; (3) utilization of glucose in the peripheral tissues.

\section{Factors Regulating Blood Sugar Levels}

\section{A. ENDOCRINE CONTROL}

\section{THE PANCREAS}

(a) Insulin. (i) Insulin facilitates the transport of glucose through cellular membranes, particularly in muscle cells. It also facilitates the further phosphorylation of glucose inside the cells, thus introducing it into the cellular metabolic machinery where it will be utilized. From this, it is obvious that insulin favours the utilization of glucose by the tissue.

(ii) Insulin increases the building up of proteins from amino acids. ${ }^{17}$ This action is independent of the action of the hormone on carbohydrate metabolism, and occurs within the cells to facilitate the incorporation of amino acids into proteins after these acids have been formed from their various precursors. ${ }^{18}$

(iii) Insulin produces marked increase in fat synthesis ${ }^{19}$ when added to isolated adipose tissue. This action is presumably secondary to enhanced glucose utilization. It has been found ${ }^{20}$ that, in adipose tissue, insulin stimulates glucose oxidation via the pentose phosphate pathway to a much greater extent than via the glycolytic pathways. The preponderance towards the pentose phosphate pathway will, as was mentioned before, favour the formation of reduced triphosphopyridine nucleotide (TPNH), which is required in the process of fat synthesis. ${ }^{21}$

(iv) Insulin favours glycogenesis in the liver and reduces glucose production from glycogen (glycogenolysis). This action of insulin in the liver will be discussed later in more detail.

(b) Glucagon. This is another hormone found in the pancreatic islets. The precise site of origin of this hormone is not yet fully established, although it is thought that it may be the alpha cells of the pancreas. ${ }^{22}$ The most important effects of glucogen administration are a rise in the concentration of blood glucose and an immediate decrease in liver glycogen (glyoogenolysis). The glycogenolytic effect of glucogen is similar to that of adrenaline except for the fact that glucogen hyperglycaemia is not blocked by dihydroergotamine. ${ }^{23}$ 


\section{THE PITUITARY}

Three of the anterior pituitary hormones are known to influence carbohydrate metabelism.

(a) Growth hormone. ${ }^{24}$ The role of growth hormones in normal carbohydrate metabolism is to conserve glucose, glycogen, and blood sugar in the fasting state. Thus, it is of interest that fasting stimulates, and glucose feeding inhibits, the rate of production of this hormone. In summary, the growth hormone: (i) Inhibits the hexokinase reaction. (ii) Damages the islets of Langerhan and may lead to permanent diabetes if the administration is not stopped in time. Insulin can reverse these effects it if is not withheld for a long time. (iii) Directly affects protein metabolism by promoting the incorporation of amino acids into proteins. ${ }^{25}$ (iv) Favours mobilization of fat from the periphery and increased uptake by the liver

(b) Prolactin. The exact role of this hormone is unknown, but it was found that it diminished or abolished the hypoglycaemia and hypersensitivity to insulin seen in hypophysectomized dogs. ${ }^{26}$

(c) $A C T H$. The actions of ACTH are almost identical with those of adrenocortical hormones except for the fact that ACTH stimulates lipid mobilization in vitro when added to adipose tissue. ${ }^{27}$ This action is extra-adrenal, since hydrocortisone itself does not cause lipid mobilization.

\section{ADRENOCORTICAL STEROIDS}

Adrenalectomy, like hypophysectomy, leads to hypersensitivity to insulin and reduction of blood sugar levels and glycosuria in the diabetic animal.

The adrenal cortical hormones affect carbohydrate metabolism in the following ways:

(a) They stimulate gluconeogenesis by stimulating the activity of the enzyme glutamic-pyruvic transaminase, ${ }^{28,29,30}$ facilitating the conversion of amino acids into pyruvic acid. This enzyme system is not affected by growth hormone, testosterone, or insulin. Another way of stimulating gluconeogenesis by adrenocorticoids is through increasing the activity of the enzyme fructose-1,6-diphosphatase, which favours a step in the process of building up glucose from pyruvic acid via the Meyerhof pathway. ${ }^{31}$

(b) They inhibit the hexokinase reaction and under certain conditions the mechanism responsible for the transport of glucose across the cell membrane..$^{32}$

(c) Cortisone reduces glycogenolysis by inhibiting the enzyme phosphorylase, which converts glycogen to glucose-6-phosphate.

(d) Adrenocortical steroids, as well as the growth hormone in the plasma, antagonize insulin in diabetic animals.

4. EPINEPHRINE

(a) Epinephrine mobilizes liver glycogen. It favours the conversion of inactive phosphorylase to the active form..$^{33}$

(b) It mobilizes fat from adipose tissue in vitro. ${ }^{34}$

5. THYROID

(a) Thyroid hormone accelerates the rate of absorption of sugar from the alimentary tract. In hyperthyroidism there is usually alimentary hyperglycaemia 
and occasional glycosuria, while hypothyroidism is often associated with a flat glucose tolerance curve.

(b) Thyroid hormones favour glycogenolysis and in hyperthyroidism the liver glycogen is depleted.

(c) The superimposition of hyperthyroidism on diabetes leads to marked deterioration of the diabetic condition, while the superimposition of myxoedema results in lowering of the severity of the diabetes. ${ }^{35}$

\section{B. ROLE OF THE LIVER}

The liver plays a very important role in maintaining the blood glucose level within a narrow range, regardless of whether the individual has just had a meal or is in the interval between meals. Thus when the blood glucose level is high, as after a meal, the liver will remove glucose from the blood; while if the blood glucose level is low, as occurs between meals, glucose will be produced by the liver and added to the blood. The mechanisms concerned with this fine regulatory function of the liver are fully discussed in an excellent review by Cahill et al. ${ }^{36}$ to which the reader is referred for more detailed information.

The hepatic cellular membrane is permeable to glucose or, in other words, it does not actively oppose or actively aid the transfer of glucose in one or the other direction, and it has been reported that the liver contains glucose concentrations approximately equal to those in the blood. ${ }^{37}$ It is thus obvious that the transfer of glucose across the hepatic cellular membrane is not the rate-limiting factor in the glucostatic role of the liver. Consequently, it seems reasonable to assume that the enzyme systems which control the entry and exit of glucose into and out of the metabolic machinery (rather than into and out of the cell, across the cellular membrane) actually are the rate-limiting factors in this important hepatic function.

The relationship of these enzymes is as follows (Fig. 4). Two reactions are of great importance and will be discussed in more detail.

1. After its transfer through the cell membrane from the blood, glucose has to be enzymatically phosphorylated to glucose-6-phosphate (Step 1) before it can be incorporated in the metabolic pool. The liver contains a glucokinase enzyme which is a specific hexokinase for glucose. This is the enzyme responsible for the phosphorylation of glucose and utilizes a high-energy phosphate bond from adenosine triphosphate. The glucokinase enzyme is under the influence of insulin, but is not affected by the presence of absence of adrenal or pituitary glands. Insulin given in vivo produces an increase in hepatic glucose phosphorylation, but only after a latent period of $6-12$ hours, ${ }^{38.39}$ in contradistinction to the increase in muscle glucose phosphorylation which occurs immediately after the insulin is given. ${ }^{40}$ When insulin is abruptly withdrawn hepatic glucose phosphorylation falls gradually over a period of $48-96$ hours. ${ }^{41}$ This sluggish response of the glucokinase activity to insulin may serve as a physiological buffer for abrupt changes in blood glucose levels.

2. The second important reaction is the reverse of the first one. Here, the glucose-6-phosphate is enzymatically changed into glucose with the aid of the enzyme glucose-6-phosphatase (Step 2). This enzyme is present in the liver and, 


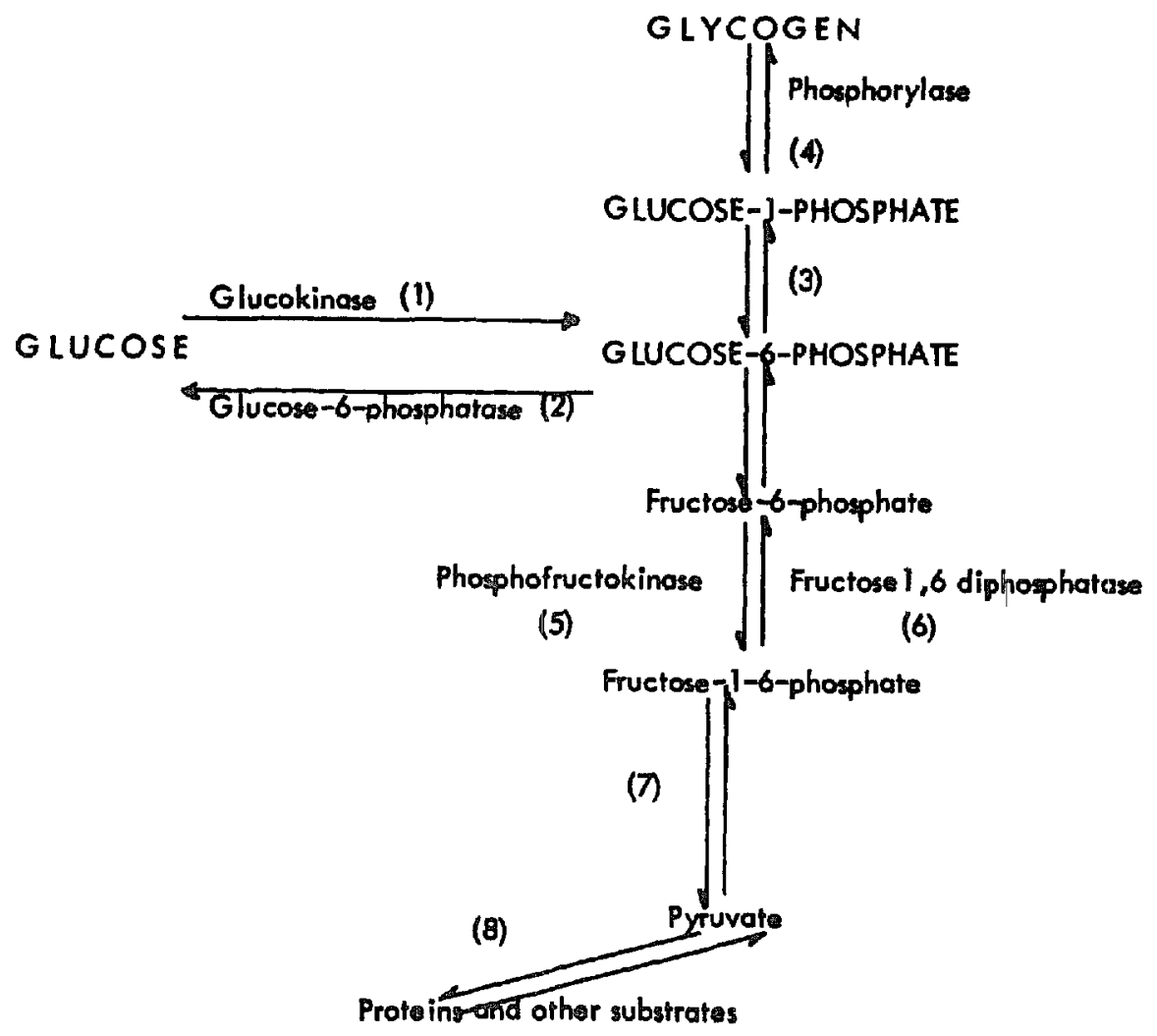

Figure 4. Hepatic carbohydrate metabolism.

to a limited extent, in the renal proximal tubules and other tissues, ${ }^{42,43,44}$ but it seems that the enzyme plays its major physiological role (dephosphorylation of glucose-6-phosphate) only in the hepatic site. It is thus clear that the rate and direction of the reaction glucose $\rightleftharpoons$ glucose- 6 -phosphate depends on the following: $(a)$ activity of glucokinase, $(b)$ activity of glucose-6-phosphatase, (c) the concentration of free glucose, $(d)$ the concentration of glucose-6-phosphate.

A steady state is achieved when both these raections occur at equal rates. A change of any of the above-mentioned four factors would alter this steady state. It must be emphasized, however, that the liver can still maintain a steady state without any change in the activity of the two enzymes, presumably through changes in the blood glucose levels, which would reflect comparable changes in the rate and direction of the reaction glucose $\rightleftharpoons$ glucose- 6 -phosphate.

FATE OF Glucose-6-Phosphate

This important substrate formed by the phosphorylation of glucose could give rise to the following:

(a) It might be dephosphorylated back to glucose under the influence of the enzyme glucose-6-phosphatase (Step 2).

(b) It might be transformed into glucose-1-phosphate (Step 3), then to glycogen (Step 4), which is stored in the liver.

(c) Glucose-6-phosphate can be dissimilated along the Embden-Meyerhof pathways. However, catabolism by glycolysis is not at all common in the liver, and it seems that there is a rate-limiting reaction that minimizes the amount of 
glucose-6-phosphate following this pathway. This rate-limiting reaction is thought to be the formation of fructose-1,6-diphosphate from fructose-6-phosphate (Step $5)^{44}$ catalyzed by phosphofructokinase.

(d) Glucose-6-phosphate, being a branch point for carbohydrate metabolism, may be dissimilated along the direct oxidative or pentose phosphate pathway.

The amount of glucose-6-phosphate destined to follow each of the abovementioned channels has been calculated as follows ${ }^{36}$ : of every sixteen molecules of glucose phosphorylated in the liver, eight are dephosphorylated by glucose-6phosphatase (Step 2), four are built up into glycogen (Step 3 and 4), and three are glycolyzed, and of these one may be oxidized via the citric acid cycle. The remaining one phosphorylated glucose molecule is oxidized via the pentose phosphate pathway.

It must be remembered that phosphorylation of glucose is not the only source of glucose-6-phosphate, and that this substrate can be built up from pyruvate, or other metabolic substrate, in the citric acid cycle by a reversal of the EmbdenMeyerhof pathway (neoglucogenesis).

Factors and Conditions Affecting the Glucostatic Function of the Liver

1. Fasting or starvation produce the following: (a) Marked decrease in hepatic glucokinase activity probably due to persistently low insulin production. (b) Increased activity of glucose-6-phosphatase ${ }^{454647}$ promoting the channelling of glucose-6-phosphate towards glucose production. (c) Increased activity of fructose-1,6-diphosphatase ${ }^{31}$ promoting gluconeogenesis (Step 6, Fig. 4). (d) The reduction of blood sugar level by itself, without changes in the activity of the enzymes involved, would favour glycogen mobilization as a result of decreasing concentration of glucose-6-phosphate.

These changes will lead to increased mobilization of glycogen, increased glucose production by the liver, and diminished formation of glucose-6-phosphate.

2. Diabetes mellitus. From the metabolic point of view, a diabetic liver is a fasting liver in which the biochemical changes are grossly exaggerated: $(a)$ There is a marked depression of the hepatic glucokinase enzyme. This may actually be the primary abnormality in the diabetic liver. (b) Doubling of the activity of the glucose-6-phosphatase enzyme.

The steady state at which both enzyme systems turn over the same number of glucose molecules is reached only at higher and higher levels of blood sugar. Thus in mild diabetes this state may occur at a blood glucose level of $250 \mathrm{mg}$. After meals the blood glucose level may go up to $350 \mathrm{mg}$. per cent and this will favour glycogen deposition in the liver, while the post-absorptive blood level may be $150 \mathrm{mg}$. per cent, which will favour glycogen mobilization.

In severe diabetes, however, this steady state may never be reached and the liver will not cease producing glucose, because with more glucose added to the blood, more and more of it will escape in the urine.

Insulin injected in the diabetic leads to the following: (1) restoration to normal activity of the glucokinase and glucose-6-phosphatase enzymes a few hours after the prompt fall in blood sugar levels; (2) reduction in the rate of gluconeogenesis after a similar lag of a few hours.

3. Adrenocorticoids. Administration of glucocorticoids to normal animals 
produces the following: $(a)$ increased gluconeogenesis through stimulation of the fructose-1,6-diphosphatase enzyme; $(b)$ increased glucose-6-phosphatase activity, probably owing to increased production of glucose-6-phosphate resulting from Step $(a)^{46,48,49}$; $(c)$ glucokinase activity not changed. ${ }^{50}$ The sum total of these changes is a rise in blood sugar levels (steroid diabetes) and marked hepatic glycogen deposition.

Bilateral adrenalectomy in the diabetic produces the following: $(a)$ interruption of gluconeogenesis; $(b)$ glucokinase activity remains at the diabetic level (depressed); (c) glucose-6-phosphatase activity is depressed from the high levels in the diabetes towards normal.

These changes explain why bilateral adrenalectomy improves the diabetit condition. ${ }^{51}$ However, it must be realized that the amelioration of the diabetic state by superimposed adrenalectomy also leaves the animal's liver unable to buffer either hypo- or hyper-glycaemia. Thus in hypoglycaemia the liver will have very little glycogen and glucose-6-phosphate to call upon to replenish the blood sugar and in hyperglycaemia the depression of the glucokinase enzyme will prevent adequate uptake of excess blood glucose by the liver.

Bilateral adrenalectomy in the nornal produces the following: (a) depressed gluconeogenesis, which leads to depletion of the glycogen contents of the liver; (b) glucokinase and glucose-6-phosphatase enzymes are nearly normal. The animal will have a normal carbohydrate metabolism, but will be unable to stand prolonged fasting because the only supply of blood sugar in this event will be the much depleted hepatic glycogen stores.

4. Adrenaline. Adrenaline injection produces immediate increase in blood glucose through enhanced hepatic glycolysis by activating the phosphorylase reaction (Fig. 4, Step 4).

5. Glucogen produces the same effects as adrenaline.

\section{Interrelationships of Glucose and Fat Metabolism ${ }^{52}$}

A great deal of work has been done on this rather involved and complicated subject in an effort to elucidate the reasons behind the changes in lipid metabolism often encountered in the diabetic, e.g., ketosis and high cholesterol levels.

1. Carbohydrate metabolism and fatty acid synthesis. There is no doubt now that the rate of glucose breakdown greatly influences lipogenesis. A high carbohydrate diet markedly stimulates the rate of fatty acid synthesis both from acetyl coenzyme $A$ and from glucose ${ }^{53,54}$ while fasting promptly depresses the rate of lipgenesis. This depression can be specifically reversed with glucose. ${ }^{55}$ Present evidence indicates that the oxidative pathways of glycolysis (pentose phosphate pathway), rather than the Embden-Meyerhof pathway, are primarily responsible for the striking effect of glucose catabolism on lipogenesis. This eflect of the oxidative pathways of glycolgesis is probably attributable to the generated reduced triphosphopyridine nucleotide TPNH which seems to be an essential hydrogen donor for at least one of the reactions involved in the process of lipogenesis.

2. Carbohydrate metabolism and fatty acid oxidation. Normal carbohydrate metabolism definitely influences fatty acid oxidation. This is thought to be achieved through at least two mechanisms: 
(a) Conversion of triglyceride to free fatty acids in the adipose tissue: thus increased glucose utilization causes a reduction in the rate of release of more esterified fatty acids from isolated slices of adipose tissue. ${ }^{56}$ Starvation or diabetes, on the other hand, results in accelerated formation of free fatty acids.

(b) Glucose must also have a direct effect on the intracellular oxidation of fatty acids to carbon dioxide, and glucose lack leads to increased rate of oxidation. The mechanisms involved in this effect are unknown.

3. Ketosis. Ketosis is a condition where there is accumulation of ketone bodies, viz., acetoacetic acid, beta-hydroxybutyric acid, and acetone. Accumulation of ketone bodies is probably due to excessive formation ${ }^{57}$ rather than diminished utilization. ${ }^{58}$

The origin of the above-mentioned ketone bodies has been shown by isotope studies to be derived from acetyl coenzyme $A$. Thus it seems reasonable to assume that conditions which increase the production or diminish the disposal of the acetyl $\mathrm{CoA}$ would eventually lead to increased formation of ketone,bodies and ketosis. In diabetes and starvation both mechanisms can occur, at least theoretically, thus contributing to the accumulation of acetyl $\mathrm{CoA}$, and subsequent ketosis.

(a) Formation of acetyl CoA. As has been mentioned before, depression of glycolysis leads to increased fatty acid mobilization and oxidation, which leads to excessive formation of the two-carbon particle (acetyl CoA).

(b) Disposal of acetyl CoA. There are two major pathways by which this may occur: (i) Final dissimilation to $\mathrm{CO}_{2}$ and water, via the citric acid cycle. Depression of glycolytic pathways will lead to inadequate supply and replacement of the fourcarbon substances (oxaloacetic acid and malic acid), which are essential for the proper functioning of the citric acid cycle. Thus in diabetes or starvation this route of disposal will be more or less blocked, leading to accumulation of acetyl CoA. (ii) Assimilation of acetyl CoA particles to form long-chain fatty acids. This pathway is also blocked, because of depression of lipogenesis (vide supra).

In summary, in diabetes and starvation all factors seem to favour the accumulation of acetyl CoA, thus leading to the excessive formation and accumulation of ketone bodies.

\section{RÉSUMÉ}

L'auteur a parlé des différentes façons dont se fait le métabolisme des hydrates de carbone: elles comprennent la voie glycolytique Embden-Meyerhof, le cycle de l'acide citrique et la voie du phosphate pentose avec quelques allusions à leurs fonctions et à leur signification.

Il a parlé ensuite du contrôle des taux du sucre sanguin en insistant particulièrement sur le rôle du foie comme organe glucostatique et sur l'interrelation entre le métabolisme des graisses et celui des hydrates de carbone.

\section{REFERENCES}

1. Marks, P. A. \& Freedman, A. D. Carbohydrate Metabolsim; Metabolic Interrelations and Control. New York State J. Med. 60: 3105 (1960).

2. Horecker, B. L. \& Mehler, A. H. Carbohydrate Metabolism. Ann. Rev. Biochem. 24: 207 (1955). 
3. Marks, P. A. A Newer Pathway of Carbohydrate Metabolism; The Pentose-Phosphate Pathways. Diabetes 5 : 276 (1956).

4. Siperstein, M. D. Inter-relationship of Glucose and Lipid Metabolism. Am. J. Med. 26: $685(1959)$.

5. Holzer, H. Carbohydrate Metabolism. Ann. Rev. Biochem. 28: 171 (1959).

6. Chance, B. \& Hess, B. Metabolic Control of Mechanisms. I. Electron Transfer in The Mammalian Cell. J. Biol. Chem. 234: 2404 (1959).

7. Cahill, G. F. Jr.; Ashmore, J.; Earl, A. S.; \& Zotru, S. Glucose Penetration into Liver. Am. J. Physiol. 192: 491 (1958).

8. Levine, R.; Goldstein, M. S.; Klein, S.; \& Húddlestun, B. The Action of Insulin on the Distribution of Galactose in Eviscerated Nephrectomized Dogs. J. Biol. Chem. 179: 985 (1949).

9. Goldstein, M. S.; Henry, W. L.; Huddlestun, B.; \& Levine, R. Action of Insulin on Transfer of Sugars Across Cell Barriers; Common Chemical Configuration of Substances Responsive to Action of the Hormone. Am. J. Physiol. 173: 207 (1953).

10. Knowlton, A. I. Diabetes Mellitus, Endocrine Relationship. New York State J. Med. 60: $3442(1960)$.

11. Harper, H. A. Review of Physiological Chemistry, 7th ed. Los Altos, Calif., Lange Medical Publication (1959).

12. Racker, E.; \& Krimsky, I. The Mechanism of Oxidation of Aldehydes by Glyceraldehyde-3Phosphate Dehydrogenase. J. Biol. Chem. 198: 731 (1952).

13. Brady, R. O.; Mamoon, A. M.; \& Stadtman, E, R. The Effects of Citrates and Co-enzyme A on Fatty Acid Metabolism. J. Biol. Chem. 22Q: 795 (1956).

14. Siperstein, M. D. Glycolytic Pathways: Their Relation to the Synthesis of Cholesterol and Fatty Acids. Diabetes 7: 181 (1958).

15. Abraham, S.; Matthes, K. J.; \& Chaikoff, I. L. Role of TPNH in Fatty Acid Synthesis From Acetate by Normal and Diabetic Rat Liver Homogenate Fraction. Biochem. et Biophys. Acta. $36: 556$ (1959).

16. Marks, P. A.; Johnson, A. B.; \& Hirschberg; E. Effect of Age on the Enzyme Activity in Erytherocytes. Proc. Nat. Acad. Sci. 44: 529 (1958).

17. KRAHL, M. E. Incorporation of $\mathrm{C}^{14}$ Amino Acids into Peptides by Normal and Diabetic Rat Tissues. Science 116:524 (1952).

18. MANChester, K. L. \& KRAHL, M. E. Effect of Insulin on the Incorporation of C ${ }^{14}$ from $\mathrm{C}^{14}$-Labelled Carboxylic Acids and Bicarbonate into the Protein of Isolated Rat Diaphragm. J. Biol. Chem. 234: 2938 (1959).

19. Winegrad, A. I. \& Renold, A. E. Studies on the Rat Adipose Tissue in Vitro. I. Effects of Insulin on the Metabolism of Glucose, Pyruvate and Acetate. J. Biol. Chem. 283: 267 (1958).

20. - Studies on Rat Adipose Tissue in Vitro. II. Effects of Insulin on the Matabolism of Specifically Labelled Glucose. J. Biol. Chem. 233: 273 (1958).

21. Langdon, R. G. The Biosynthesis of Fatty Acids in Rat Liver. J. Biol. Chem. 226: 615 (1957).

22. Lukens, F. D. W. The Pancreas, Insulin and Glucagon. Ann. Rev. Physiol. 21: 445 (1959).

23. Sirek, O. V.; Sirek, A.; \& Best, C. H. Pituitary Growth Hormone and the Question of Pancreatic Secretion of Glucagon. Am. J. Physiol. 188: 17 (1957).

24. Colowick, S. P.; Cori, G. T.; \& Slein, M. W. The Effect of Adrenal Cortex and Anterior Pituitary Extracts and Insulin on the Hexokinase Reaction. J. Biol. Chem. 168: 583 (1947).

25. Kostyo, J. L. \& KNoBil, E. The Effect of Growth Hormone in the In-Vitro Incorporation of Leucine-2-C into the Protein of Rat Diaphragm. Endocrinology 65: 395 (1959).

26. Sinkoff, M. W. \& De Bodo, R. C. Prolactin as Insulin Antagonist. Arch. Exper. Path. u. Pharmakol. Q19: 100 (1953).

27. White, J. E. \& EnGeL, F. L. Lipolytic Action of Corticotropin on Rat Adipose Tissue in Vitro. J. Clin. Invest. 37: 1556 (1958).

28. Beaton, G. H.; CURRY, D. M.; \& VEEN, M. J. Alanine-Glutamic Transaminase Activity and Protein Metabolism. Arch. Biochem. 7O: 288 (1957).

29. Rober'ts, N. R.; Rosen, F.; Budnick, L. E.; \& Nichol, C. A. An Enzymatic Basis for the Gluconeogenic Action of Hydrocortisone. Science 127: 287 (1958).

30. - Corticosteroids and Trans-aminase Activity: The Specificity of the GlutamicPyruvic Trans-aminase Response. Endocrinol. 65: 256 (1959).

31. Mokrasch, L. C.; Davidson, W. D.; \& McGilvery, R. W. The Response to Glucogenic Stress of Fructose-1,6-diphosphatase in Rabbit Liver. J. Biol. Chem. 222: 179 (1956). 
32. Park, C. R. \& Morgan, H. E. Glucose Transport and Phosphorylation in Muscles of Diabetic Animals. Diabetes 9: 250 (1960).

33. Sutherland, E. W. The Effect of the Hyperglycemic Factor and Epinepherine on Enzyme Systems of Liver and Muscle. Ann. New York Acad. Sci. 54: 693 (1951).

34. Gordon, R. S., JR. \& Cherkes, A. Production of Unesterified Fatty Acids From Isolated Rat Adipose Tissue Incubated in Vitro. Proc. Soc. Exper. Biol. \& Med. 97: 150 (1958).

35. Rupp, J. J.; Di George, A. M.; \& Paschkis, K. E. Hypothyroidism and Diabetes Mellitus. Diabetes 4: 393 (1955).

36. Cahill, G. F., Jr.; Ashmore, J ; Renold, A ; \& Hastings, A. B. Blood Glucose and the Liver. Am. J. Med. 26: 264 (1959).

37. Gey, K. F. The Concentration of Glucose in Rat Tissues. Biochem. J. 64: 145 (1956).

38. Renold, A. E.; Hastings, A. B.; Nesbett, F. B.; \& Ashmore, J. Studies on Carbohydrate Metabolism in Rat Liver Slices. IV. Biochemical Sequence of Events After Insulin Administration. J. Biol. Chem. 318: 135 (1955).

39. Krahl, M. E. The Effect of Insulin on Glucose Uptake in Muscle. Ann. New York Acad. Sci. 54: 649 (1951).

40. Stadie, W. C. Current Concepts of the Action of Insulin. Physiol. Rev. 34: 52 (1954).

41. Spiro, R. G; Ashmore, J.; \& Hastings, A. B Studies on Carbohydrate Metabolism in Rat Liver Slices. XII. Sequence of Metabolic Events Following Acute Insulin Deprivation. J. Biol. Chem 290: 761 (1958).

42. Chrquorne, A. D. Further Studies on the Histochemistry of Glucose-6-Phosphatase. J. Histochem. \& Cytochem. 3: 471 (1955).

43. Weber, G. \& Cantero, A. Glucose-6-Phosphatase Activity in Normal, Precancerous and Neoplastic Tissues. Cancer Res. 15: 105 (1955).

44. Orson, R. E. Oxidation of $\mathrm{C}^{14}$-Labelled Carbohydrate Intermediates in Tumor and Normal Tissues. Cancer Res. 11:571 (1951).

45. Ashmore, J ; Hastings, A. B.; \& Nesbett, F. B. The Effect of Diabetes and Fasting on Liver Glucose-6-Phosphatase. Proc. Nat. Acad. Sci. 40:673 (1954).

46. Ashmore, J.; Hastings, A. B.; Neshett, F. B.; \& Renold, A. E. Studies on Carbohydrate Metabolism in Rat Liver Slices: VI. Hormonal Factors Influencing Glucose-6-Phosphatase. J. Biol. Chem. 218: 77 (1956).

47. Weber, G. \& Cantero, A. Glucose-6-Phosphatase Studies in Fasting. Science 120: 851 (1954),

48. Weber, G.; Allard, C.; De Lamirande, G.; \& Cantero, A. Liver Glucose-6-Phosphatase Activity and Intracellular Distribution After Cortisone Administration. Endocrinology, $58: 40(1956)$.

49. Weber, G. \& Cantero, A. Studies on Hormonal Factors Influencing Hepatic Glucose-6Phosphatase. Endocrinology 61: 701 (1957).

50. Ashmore, J.; Cahill, G. F., Jr.; Hillman, R.; \& Renold, A. E. Adrenal Cortical Regulation of Hepatic Glucose Metabolism. Endocrinology 62: 621 (1958).

51. Hartman, F. A. \& Brownell, K. A. Relation of Adrenals to Diabetes. Proc. Soc. Exper. Biol. \& Med. 31: 834 (1934).

52. Siperstein, M. D. Inter-relationship of Glucose and Lipid Metabolism. Am. J. Med. 26: 685 (1959).

53. Tepperman, J. \& Tepperman, H. M. Effects of Antecedent Food Intake Pattern on Hepatic Lipogenesis. Am. J. Physiol. 198: 55 (1958).

54. Masoro, E. J.; Chaikoff, I. L.; Chernick, S. S.; \& Felts, J. M. Previous Nutritional State and Glucose Conversion to Fatty Acids in Liver Slices. J. Biol. Chem. 185: 845 (1950).

55. Masri, M. S.; Lyon, I.; \& Chaikoff, I. L. Nature of the Stimulating Action of Insulin on Lipogenesis from Acetate in Fasted Rat Liver. J. Biol. Chem. 197: 621 (1952).

56. Gordon, R. S., Jr. \& Cherkes, A. Production of Unesterified Fatty Acids From Isolated Rat Adipose Tissue in Vitro. Proc. Soc. Exper. Biol. and Med. 97: 150 (1958).

57. Weinhouse, S.; Millington, R. H.; \& Friedman, B. The Effect of Carbohydrate on the Oxidation of Fatty Acids by Liver Slices. J. Biol. Chem. 181: 489 (1949).

58. Banting, F. G \& Best, C. H. The Internal Secretion of the Pancreas. J. Lab. \& Clin Med. $7: 251(1922)$. 